\title{
Effectiveness of needle and syringe programmes for preventing HIV transmission
}

\author{
Margaret MacDonald ${ }^{\mathrm{a}, *}$, Matthew Law ${ }^{\mathrm{a}}$, John Kaldor ${ }^{\mathrm{a}}$, Jim Hales ${ }^{\mathrm{b}, 1}$, Gregory J. Dore ${ }^{\mathrm{a}}$ \\ ${ }^{a}$ National Centre in HIV Epidemiology and Clinical Research, University of NSW Level 2, 376 Victoria Street, Darlinghurst, NSW 2010, Australia \\ ${ }^{\mathrm{b}}$ Health Outcomes International Pty. Ltd., P.O. Box 1038, Kent Town, SA 5071, Australia
}

Received 1 December 2002; received in revised form 15 March 2003; accepted 2 July 2003

\begin{abstract}
Objective: To examine the effectiveness of needle and syringe programmes (NSPs) in preventing HIV transmission among injecting drug users (IDUs).

Methods: An ecological study design was used to determine change in HIV prevalence among injecting drug users between cities with and without NSPs. Several data sources, such as electronic journal databases, surveillance reports, websites, and index review of relevant journals, were used to identify studies of HIV seroprevalence among IDUs, and presence of NSPs. The rate of change in HIV prevalence was estimated by regression analysis.

Results: There were 778 years of data from 99 cities globally included in the analysis. HIV prevalence decreased by $18.6 \%$ per annum in cities that introduce NSPs, and increased by $8.1 \%$ in cities that had never introduced NSPs (mean difference $-24.7 \%$ [95\% CI: $-43.8,0.5 \%$, $P=0.06)$. The mean difference was $-33 \%$ when comparison was weighted to one over the variance of the regression estimator $(29 \%$ decrease in cities with NSPs and 5\% increase in cities without NSPs, $P<0.001)$. When analysis was restricted to cities with first HIV seroprevalence less than $10 \%$, the average annual change in seroprevalence was $18 \%$ lower in cities with NSPs $(P=0.03)$.

Conclusions: Despite the inherent limitations within an ecological study design, the study provides additional evidence that NSPs reduce transmission of HIV infection. The rapid spread of HIV among IDU populations and increasing rates of injecting in many countries calls for scaling up of NSPs as well as other harm reduction strategies.
\end{abstract}

Crown Copyright (C) 2003 Published by Elsevier B.V. All rights reserved.

Keywords: Syringe exchange programmes; HIV prevention; Australia

\section{Introduction}

Measures to prevent HIV infection among people who inject drugs generally focus on preventing blood contact during injection by reducing injection or promoting use of sterile equipment when injecting. Consequently, needle and syringe programmes (NSPs) are a key strategy for preventing transmission of HIV infection in many developed countries (Drucker, Lurie, Wodak, \& Alcabes 1998). In some countries, implementation has often been limited by uncertainty about their effectiveness.

Randomised trials of the effectiveness of NSPs in preventing HIV transmission have not been conducted. It is lo-

\footnotetext{
* Corresponding author. Fax: +61-2-9332-1837.

E-mail addresses: mmacdonald@nchecr.unsw.edu.au (M. MacDonald), jim@hoi.com.au (J. Hales)

${ }^{1}$ Fax: +61-8-8363-9011.
}

gistically difficult to randomly allocate access to NSPs, although community randomisation is feasible. However, because of the strong biological plausibility of avoiding HIV infection by using a sterile syringe for injection, there are grave ethical concerns attached to randomised trials of this nature.

Several studies have assessed the impact of NSPs on self-reported risk behaviours, in particular use of sterile syringes or re-use of one's own syringe since NSP introduction (reviewed in Drucker et al., 1998). A few studies have compared HIV incidence or prevalence between participants and non-participants of NSPs (Bruneau, Lamothe, \& Franco 1997; Des Jarlais, Hagan, \& Friedman, 1996; Hagen, McGough, \& Thiede 1999; Van Ameijden, Van den Hoek, Mientjes, \& Coutinhovan, 1993). One study compared NSP implementation in countries with sustained low HIV prevalence to those with high HIV prevalence (Des Jarlais et al., 1995). Another used an ecological study design to compare 
changes in HIV prevalence in cities with and without NSPs (Hurley, Jolley, \& Kaldor, 1997).

Data generally, but not always, show NSPs to be effective in preventing HIV transmission. Based on the previous ecological study methodology (Hurley et al., 1997), we have examined the effectiveness of NSPs in reducing HIV infection because several countries have introduced NSPs since the initial study was carried out in the mid-1990s.

\section{Methods}

Comparison of HIV infection among studies of injecting drug users (IDUs) in cities with and without NSPs was made using an ecological study design. Data on HIV seroprevalence and presence or absence of NSPs were identified to the end of 2000. HIV prevalence estimates based on saliva sampling were also included. NSPs were defined as programmes distributing needles and syringes. The operational characteristics of NSPs vary widely both within and between cities and countries. However, for the purposes of this study NSPs were included irrespective of whether they provided syringes free or with minimal charge, whether they operated from a fixed or mobile site, whether return of a used syringe was mandatory, or the range of other HIV prevention and treatment services provided.

Several sources were used to identify reports of HIV prevalence among injecting drug users and implementation of NSPs. Three electronic databases, Medline, Embase and Current Contents, were searched from January 1984 to June 2001. Additional studies were obtained from country specific surveillance reports, the HIV/AIDS Surveillance Database (US Census Bureau \& UNAIDS, 2000), relevant websites, and through review of the index of relevant journals. Email contact was also made with key researchers in most countries.

Studies with sample size of at least 50 were included. Cities with HIV prevalence studies were only included if HIV was measured among injecting drug users in 2 or more calendar years. Studies of HIV among incarcerated injecting drug users were excluded because very few countries provided NSPs during imprisonment. Studies reported in journals published in languages other than English were only included if all required data points and sufficient information to determine whether the study was suitable for inclusion were provided in the abstract. References for seroprevalence data used in the analysis are available on request.

Number of injectors tested per calendar year, percentage with HIV antibody, presence or absence of NSP, and recruitment site were recorded for all studies. If studies reported data aggregated for more than 1 calendar year, the mid-point of the study period was used as the survey date.

Analyses compared city specific change in HIV prevalence in cities with NSPs to those without NSPs at the time of the surveys. The annual rate of change of HIV prevalence was estimated for each city by fitting a regression line on a logit scale, with calendar years centred to 1990. Annual rate of change of HIV prevalence was also estimated using regression weighted comparison of cities with and without NSPs according to one over the variance of the regression estimator to allow comparison with the previous ecological study (Hurley et al., 1997). The effect of NSPs was assessed by comparing the annual rate of change in HIV seroprevalence in cities that had ever introduced NSPs with cities that had never introduced NSPs.

Analyses of HIV seroprevalence were performed comparing all cities, and also in the subset of cities with an initial HIV seroprevalence of less than $10 \%$ that had results from at least three surveys available over at least 3 years. Analyses were repeated using regressions weighted according to survey sample size, and excluding cities in developing countries.

\section{Results}

\section{HIV seroprevalence}

There were 778 calendar years of data from 99 cities with HIV seroprevalence measurements from more than 1 year and information on NSP implementation. Studies were from 63 cities without NSPs and 36 cities with NSPs (Table 1). HIV prevalence ranged from 0 to $79 \%$ at the first data point for each city (median 18). Studies with first HIV prevalence of $10 \%$ or less were available from 19 cities without and 25 cities with NSPs. To illustrate the fitting procedure, the fitted regression lines and the reported HIV seroprevalence survey results are shown for two typical cities (Milan, Italy and Sydney, Australia) in Fig. 1a and b, respectively.

The overall comparison of annual rates of change of HIV seroprevalence in cities that never introduced NSPs with cities that did introduce NSPs are summarised in Table 2. Cities that introduced NSPs had a mean annual $18.6 \%$ decrease in HIV seroprevalence, compared with a mean annual $8.1 \%$ increase in HIV seroprevalence in cities that had never introduced NSPs (mean difference $-24.7 \%, P=0.06$ ). The mean difference was $32.7 \%$ when comparison was weighted to one over the variance of the regression estimator $(29.2 \%$ decrease in cities with NSPs and $5.1 \%$ increase in cities without NSPs, $P<0.001$ ).

In cities with an initial HIV prevalence less than $10 \%$ and with sero-surveys over a period of at least 3 years, the mean annual weighted increase in HIV prevalence was $32.1 \%$ in cities that did not introduced NSPs, compared with a mean annual decrease of $7.8 \%$ in cities with NSPs (weighted mean difference $-18.4 \%, P=0.03$ ).

Variability of the point estimate was markedly reduced and statistical significance markedly increased when the analyses for all cities and cities with HIV prevalence less than $10 \%$ were weighted according to one over the regression estimate. There was little change in the point estimate using weighted analysis. Other analyses, using regressions 


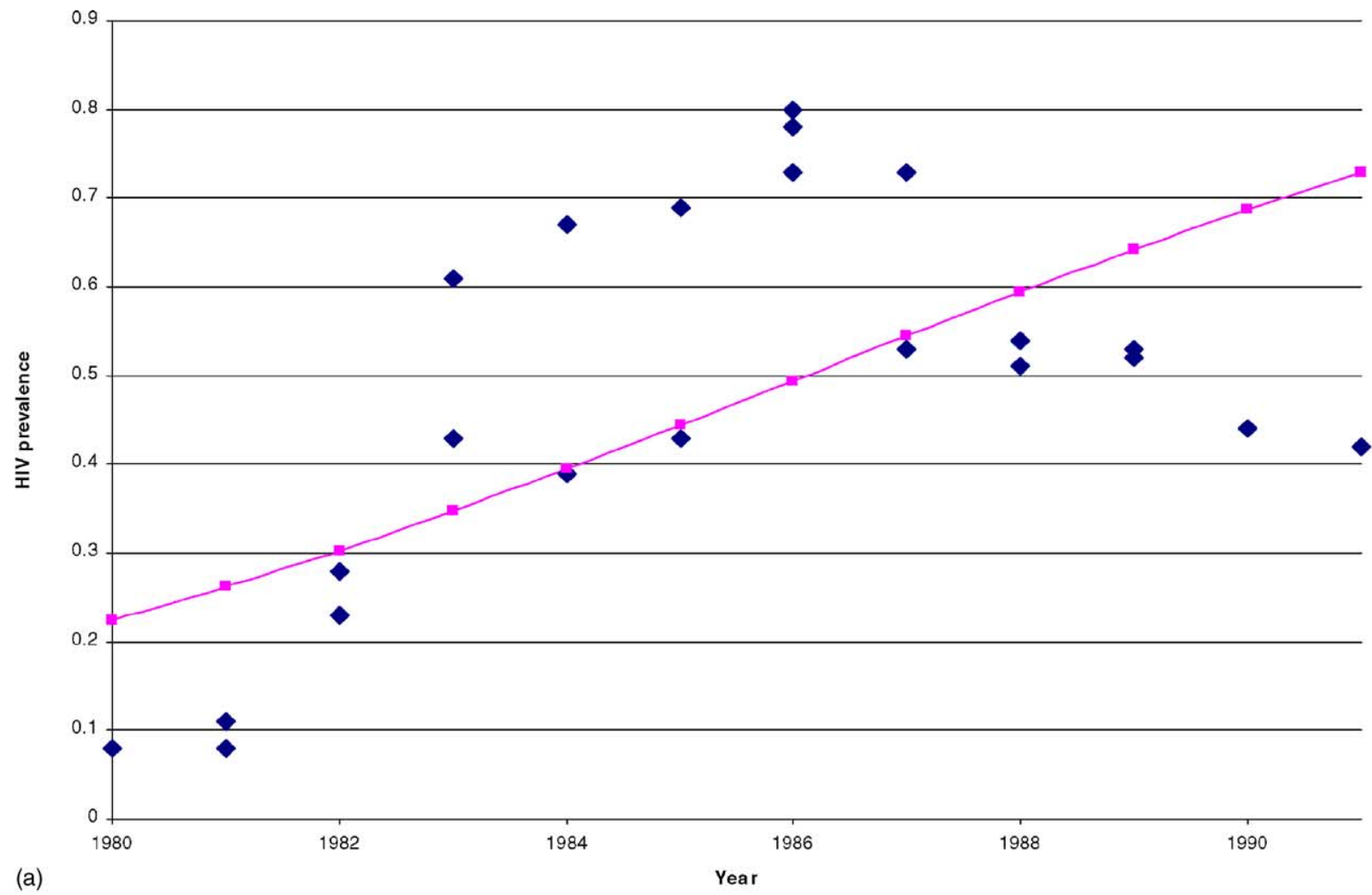

(a)

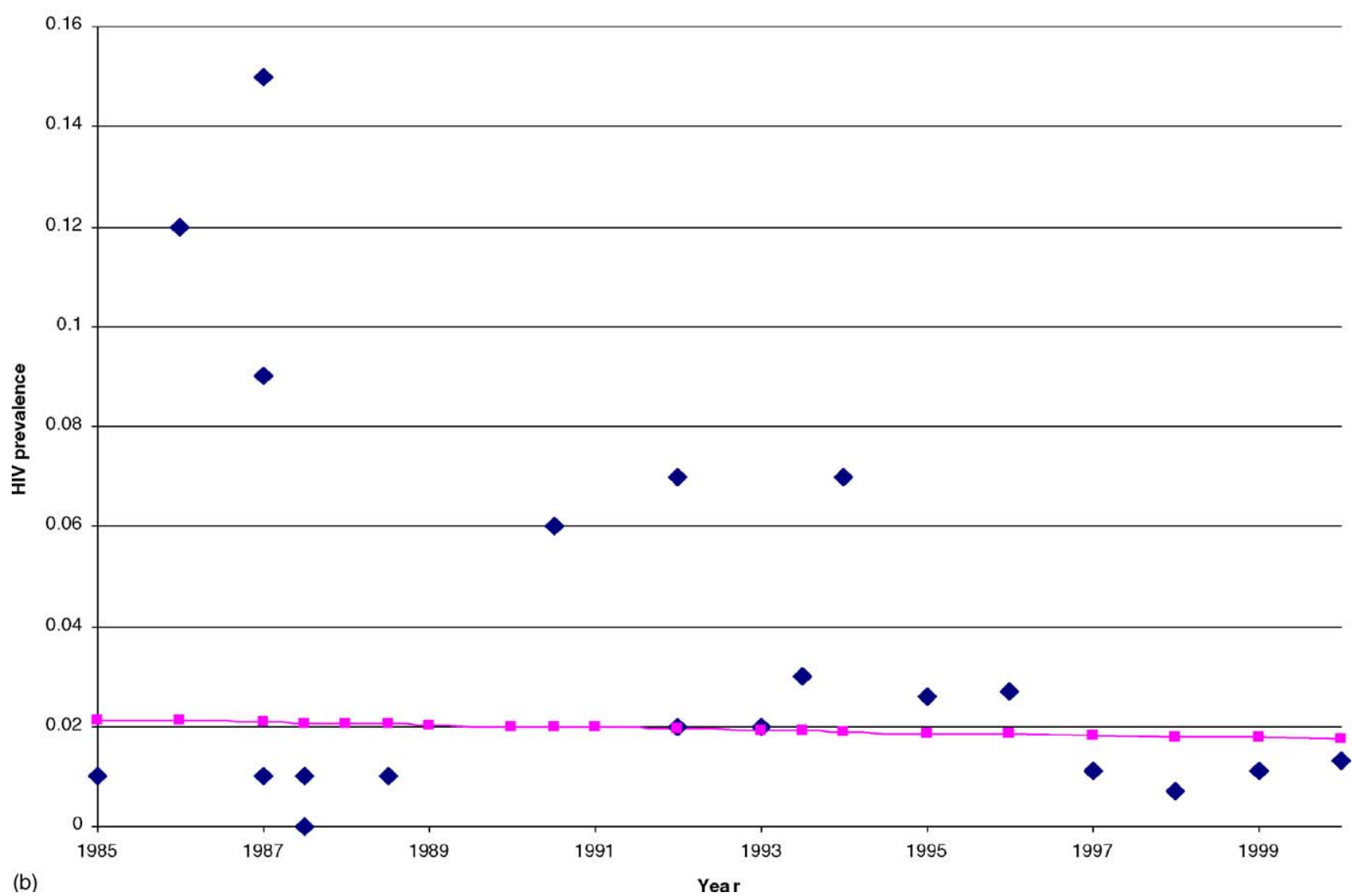

Fig. 1. (a) HIV seroprevalence in injecting drug users per year of survey for a city without NSP, Milan, Italy. (Lines represent fitted values from the logistic regression model.) (b) HIV seroprevalence in injecting drug users per year of survey for a city with NSP, Sydney, Australia. (Lines represent fitted values from the logistic regression model.) 
Table 1

Location of studies and sites of recruitment for cities with at least two HIV prevalence studies according to NSP status

\begin{tabular}{|c|c|c|}
\hline Location of studies & $\begin{array}{l}\text { Number of cities } \\
\text { without NSP }\end{array}$ & $\begin{array}{l}\text { Number of cities } \\
\text { with NSP }\end{array}$ \\
\hline \multicolumn{3}{|l|}{ Asia } \\
\hline China & 3 & 0 \\
\hline India & 1 & 1 \\
\hline Malaysia & 4 & 0 \\
\hline Myanmar & 4 & 0 \\
\hline Nepal & 0 & 1 \\
\hline Thailand & 18 & 2 \\
\hline Vietnam & 0 & 1 \\
\hline Australia & 0 & 10 \\
\hline Canada & 0 & 3 \\
\hline \multicolumn{3}{|l|}{ Europe } \\
\hline Austria & 0 & 1 \\
\hline Czech Republic & 1 & 0 \\
\hline Denmark & 0 & 1 \\
\hline France & 0 & 1 \\
\hline Germany & 0 & 1 \\
\hline Greece & 0 & 1 \\
\hline Israel & 1 & 0 \\
\hline Italy & 10 & 0 \\
\hline The Netherlands & 0 & 2 \\
\hline Spain & 3 & 0 \\
\hline Switzerland & 0 & 1 \\
\hline \multicolumn{3}{|l|}{ South America } \\
\hline Argentina & 0 & 1 \\
\hline Brazil & 5 & 0 \\
\hline United Kingdom & 0 & 5 \\
\hline United States & 13 & 4 \\
\hline Total cities & 63 & 36 \\
\hline \multicolumn{3}{|l|}{ Recruitment sites } \\
\hline Forensic medicine institutes & 0 & 4 \\
\hline Detoxification/rehabilitation & 226 & 19 \\
\hline Drug treatment agency & 95 & 80 \\
\hline Entry to treatment & 33 & 17 \\
\hline Field \& snowball & 16 & 32 \\
\hline Health service & 0 & 2 \\
\hline HIV testing centre & 6 & 17 \\
\hline Infectious diseases hospital & 14 & 1 \\
\hline Multiple sites & 15 & 67 \\
\hline NSP/pharmacy & 0 & 62 \\
\hline Sexual health clinics & 4 & 14 \\
\hline Other/not reported & 26 & 28 \\
\hline Total studies & 435 & 342 \\
\hline
\end{tabular}

weighted according to survey sample size, and also excluding cities in developing countries, gave similar results (data not presented).

\section{Discussion}

On average, HIV seroprevalence decreased in studies of injecting drug users in cities with NSPs whereas in studies from cities without NSPs, HIV seroprevalence increased. Similar results were obtained when analysis was restricted to cities with initial HIV prevalence of less than $10 \%$, weighted according to survey sample size or when cities in developing countries were excluded.

There are several limitations associated with the ecological study design that should be considered when interpreting the findings from these studies. Seroprevalence data used in the analyses were collected according to different protocols and in diverse populations. It is possible that recruitment through NSPs might provide access to less dependent injectors than those recruited through treatment services. However, there is also some evidence to suggest that some NSPs attract injectors with high levels of HIV risk behaviour (Hagen et al., 1999; Hahn, Vranizan, \& Moss, 1997; Miller, Tyndall, Spittal, Li, \& Palepu, 2002). Because cities were selected for analysis by the existence of published HIV serological surveys, bias may have been introduced by the decision to do a survey in a particular city at a particular time.

Data on NSPs used in the analyses were based on presence or absence of NSPs rather than on the extent and uptake of these services. Given the positive findings, that is, that presence of NSPs results in a decreased HIV prevalence, then it is likely that HIV prevalence would be lower in cities where availability and uptake of NSPs is extensive compared to cities with limited uptake. In addition, it was not possible to separate the effects of implementation of NSPs from the other HIV prevention strategies such as pharmacy availability of syringes and methadone maintenance treatment (Fischer, Rehm, \& Blitz-Miller, 2000). The excess risk of HIV in people who inject drugs is not due solely to sharing needles as other injecting practices and sexual behaviour patterns increase HIV risk.

It is also possible that HIV seroprevalence may have remained low in some of the cities with NSPs, irrespective of their introduction. If NSPs decrease the incidence of HIV, the rate of increase in seroprevalence should decrease, although the seroprevalence itself may not decrease, at least initially. The annual mean decrease in HIV seroprevalence was much lower in the repeated study, around half, than in the original paper (Hurley et al., 1997) possibly due to the longer time period represented by the studies.

NSPs influence HIV transmission by increasing use of sterile syringes for injection and lowering the rate of syringe sharing thereby reducing contact with the virus. In many settings, NSPs also provide condoms, education about minimising risk and referrals to drug treatment centres. Even though the difference in rate of change of HIV seroprevalence between cities with and without NSPs may not be due solely to NSPs, the study provides strong evidence that NSPs reduce the spread of HIV infection. Consequently, the rapid spread of HIV among IDU populations and increasing rates of injecting in many countries calls for scaling up of NSPs as well as other harm reduction strategies. 
Table 2

Estimated annual rate of change in HIV seroprevalence according to weighting of analysis and sample selection for cities without and with NSPs

\begin{tabular}{|c|c|c|}
\hline Weighting of analysis/sample selection & Cities without NSPs & Cities with NSPs \\
\hline \multicolumn{3}{|l|}{ No weighting of analysis } \\
\hline \multicolumn{3}{|l|}{ All cities } \\
\hline Number & 63 & 36 \\
\hline Mean $(95 \%$ CI $)$ & $8.1 \%(-2.8,20.1 \%)$ & $-18.6 \%(-42.6,15.3 \%)$ \\
\hline Mean difference $(95 \% \mathrm{CI})$ & $-24.7 \%(-43.8,0.5 \%), P=0.057$ & \\
\hline \multicolumn{3}{|c|}{ Cities with initial HIV prevalence $<10 \%, 3$ calendar years of data } \\
\hline Number & 19 & 25 \\
\hline Mean $95 \%$ CI & $28.6 \%(-4.9,73.8 \%)$ & $-4.0 \%(-28.5,29.0 \%)$ \\
\hline Mean difference $(95 \% \mathrm{CI})$ & $-25.3 \%(-50.8,13.3 \%), P=0.165$ & \\
\hline \multicolumn{3}{|c|}{ NSP comparison weighted to one over the variance of the regression estimator } \\
\hline \multicolumn{3}{|l|}{ All cities } \\
\hline Number & 63 & 36 \\
\hline Mean $(95 \% \mathrm{CI})$ & $5.1 \%(1.4,9.1 \%)$ & $-29.2 \%(-30.8,-27.6 \%)$ \\
\hline Mean difference $(95 \% \mathrm{CI})$ & $-32.7 \%(-37.5,-27.6 \%), P=<0.001$ & \\
\hline \multicolumn{3}{|c|}{ Cities with initial HIV prevalence $<10 \%$ and 3 calendar years of data } \\
\hline Number & 19 & 25 \\
\hline Mean & $32.1 \%$ & 7.8 \\
\hline $95 \% \mathrm{CI}$ & $(22.1,42.8 \%)$ & $(-4.8,22.0 \%)$ \\
\hline Mean difference $(95 \% \mathrm{CI})$ & $-18.4 \%(-32.0,-2.0 \%), P=0.030$ & \\
\hline
\end{tabular}

\section{Acknowledgements}

The authors would like to acknowledge the contribution of Jialun Zhou who helped locate many of the articles and the many researchers internationally who provided data and checked the data points for their country. The National Centre in HIV Epidemiology and Clinical Research is supported by the Australian Department of Health and Ageing through the Australian National Council on AIDS, Hepatitis C and Related Diseases and its Clinical Trials and Research Committee. The Return on Investments from NSP Project for which the data presented in this paper were obtained was also funded by the Australian Department of Health and Ageing and is the property of the Australian Government (Commonwealth of Australia (C) 2002).

\section{References}

Bruneau, J., Lamothe, F., \& Franco, E. (1997). High rates of HIV infection among injecting drug users participating in needle exchange programs in Montreal: Results of a cohort study. American Journal of Epidemiology, 145, 994-1002.

Des Jarlais, D. C., Hagan, H. R., \& Friedman, S. R. et al. (1995). Maintaining low HIV seroprevalences in populations of injecting drug users. The Journal of the American Medical Association, 274, 12261231.
Des Jarlais, D. C., Marmor, M., \& Paone, D. et al. (1996). HIV incidence among injecting drug users in New York City syringe-exchange programs. Lancet, 348, 987-991.

Drucker, E., Lurie, P., Wodak, A., \& Alcabes, P. (1998). Measuring harm reduction: The effects of needle and syringe exchange programs and methadone maintenance on the ecology of HIV. AIDS, 12(Suppl. A), S217-S230.

Fischer, B., Rehm, J., \& Blitz-Miller, T. (2000). Injection drug use and preventative measures: A comparison of Canadian and Western European jurisdictions over time. Canadian Medical Association Journal, 162, 1709-1713.

Hagen, H., McGough, J. P., \& Thiede, H. et al. (1999). Syringe exchange and risk of infection with hepatitis B and C viruses. American Journal of Epidemiology, 149, 203-213.

Hahn, J. A., Vranizan, K. M., \& Moss, A. R. (1997). Who uses needle exchange? A study of injection drug users in treatment in San Francisco, 1989-1990. Journal of Acquired Immune Deficiency Syndromes and Human Retrovirology, 15(2), 157-164.

Hurley, S. F., Jolley, D. J., \& Kaldor, J. M. (1997). Effectiveness of needle-exchange programmes for prevention of HIV infection. Lancet, $349,1797-1800$.

Miller, C. L., Tyndall, M., Spittal, P., Li, K., \& Palepu, A. et al. (2002). Risk-taking behaviours among injecting drug users who obtain syringes from pharmacies, fixed sites, and mobile van needle exchanges. Journal of Urban Health, 79(2), 257-265.

US Census Bureau UNAIDS Collaborating Centre. (2000). HIV/AIDS Surveillance Data Base. Health Studies Branch, International Programs Center, Population Division, U.S. Census Bureau, Washington.

Van Ameijden, E. J. C., Van den Hoek, J. A. R., Mientjes, G. H. C., \& Coutinho, R. A. (1993). A longitudinal study on the incidence and transmission patterns of HIV, HBV and HCV infection among drug users in Amsterdam. European Journal of Epidemiology, 9, 225-262. 